\title{
Etanercept: therapeutic use in patients with rheumatoid arthritis
}

\author{
Leslie Garrison, Neil D McDonnell
}

\begin{abstract}
Tumour necrosis factor (TNF) plays a central part in the pathophysiology of rheumatoid arthritis (RA). TNF initiates signal transduction by interacting with surface bound TNF receptors. Soluble tumour necrosis factor receptors (sTNFRs) act as natural inhibitors of TNF activity. Etanercept, recombinant p75 sTNFR:Fc fusion protein, has received approval from the US Food and Drug Administration for patients with RA and juvenile RA (JRA) who have failed treatment with at least one other drug. Etanercept has demonstrated excellent safety and efficacy in large scale, randomised, double blind, placebo controlled trials of patients with RA and JRA who are refractory to other disease modifying antirheumatic drugs. The therapeutic effects mediated by etanercept are rapid and sustained. Combining etanercept with methotrexate was found to be safe and more effective than treatment with methotrexate alone in the treatment of RA. These clinical findings demonstrate that etanercept can result in symptomatic improvement in patients with RA and JRA. Etanercept is an important new addition to the treatment of these diseases. (Ann Rheum Dis 1999;58:(Suppl I) I65-I69)
\end{abstract}

Tumour necrosis factor (TNF) is known to play a central part in the pathogenesis of rheumatoid arthritis (RA). As the importance of TNF in RA began to be understood, the intriguing possibility emerged that blocking the activity of TNF might improve RA symptoms and perhaps even slow disease progression. In the past few years, biological response modifiers capable of neutralising TNF have been developed and tested in patients with RA. One of these agents, etanercept, has recently been approved by the US Food and Drug Administration (FDA) for patients with RA or juvenile rheumatoid arthritis (JRA) who have failed treatment with at least one other disease modifying drug.

\section{TNF plays a central part in the} pathophysiology of RA

Immunex Corporation, Seattle, WA, USA

Correspondence to: Dr N D McDonnell, Immunex Corporation, 51 University Street, Seattle, WA 98101 , USA.
TNF is a proinflammatory cytokine that is primarily produced by monocytes, macrophages, and lymphocytes. ${ }^{1-3}$ Bioactive soluble TNF is a homotrimer composed of three identical 17 $\mathrm{kDa}$ TNF molecules. ${ }^{4} \mathrm{TNF}$ must engage two or more membrane bound TNFRs to initiate intracellular signal transduction. ${ }^{3}$
The role of TNF in arthritis has been shown in mice transgenic for human TNF, in which synovitis and destructive arthritis spontaneously develop. ${ }^{5}$ Additionally, TNF is found in high concentrations in the synovium and synovial fluid of patients with $\mathrm{RA}^{26-11}$; the level of TNF in the synovium correlates with the degree of synovitis and bone erosions. ${ }^{6}{ }^{12}$

TNF triggers several important events that lead to the synovitis and tissue destruction exhibited in RA. It causes proliferation of synoviocytes, production of other proinflammatory cytokines such as interleukin 1 (IL1), interleukin 6 (IL6), and GM-CSF; induces production of metalloproteinases such as collagenase and stromelysin, and increases expression of adhesion molecules. In addition, prostaglandin $\mathrm{E}_{2}$ production by synoviocytes is increased, which, in conjunction with IL1, IL6, and TNF itself, increase proliferation and activity of osteoclasts, leading to destruction of bone. ${ }^{13-16}$

In addition to $\mathrm{TNF}$, other proinflammatory cytokines, most importantly IL1, are thought to be involved in the pathogenesis of RA. As previously mentioned, TNF induces the expression of both IL1 and IL6. ${ }^{17-19}$ In contrast, IL1 stimulates IL6 production, but seems to be a less potent stimulator of TNF production. ${ }^{18}$

Soluble TNF receptors: natural TNF inhibitors

Although the concept of blocking TNF activity has been considered since the mid-1980s, the discovery that membrane associated TNFRs existed in soluble forms that retained ligand binding capacity led to the relatively recent development of this novel approach for neutralising TNF. sTNFR based agents have several advantages as a means of inhibiting TNF activity. sTNFR based compounds do not require non-human amino acid sequences. This reduces their antigenic potential and minimises the likelihood that humans treated with these agents will develop antibodies against them that could potentially interfere with their therapeutic effects. Furthermore, sTNFR based agents effectively neutralise circulating TNF molecules. This distinguishes them from receptor antagonists, which prevent activity by interacting with the receptor but leave the active ligand in circulation.

There are two distinct TNF receptor molecules that bind TNF with comparable affinities, the p55 or type 1 TNFR and the p75 or type 2 TNFR. ${ }^{20}$ Soluble forms of both receptors have been identified. ${ }^{21}$ These sTNFRs can neutralise TNF activity both in vivo and in vitro, and both are believed to act as 

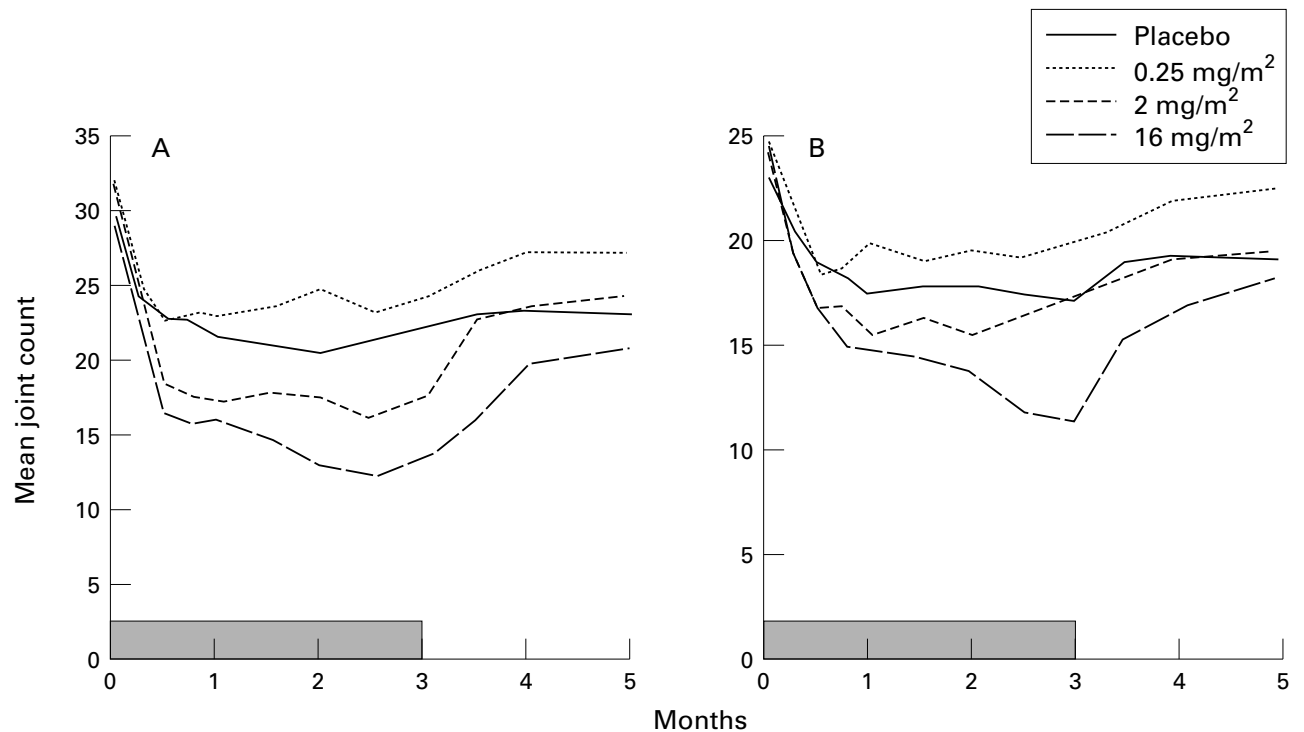

Figure 1 Effect of treatment on tender and swollen joint counts in phase II trial. (A) Mean tender joint count. (B) Mean swollen joint count. Shaded bars represent the treatment period.

natural inhibitors of TNF activity. ${ }^{21-23}$ The levels of sTNFRs are often markedly increased in the sera and synovial fluid of RA patients..$^{23-25}$ However, it seems that an imbalance between TNF and sTNFR expression contributes to the perpetuation of inflammation and subsequent joint destruction in patients with RA. ${ }^{23}$

\section{Development of etanercept for clinical use}

Cloning of the TNFR genes has allowed the expression of recombinant forms of sTNFRs. Etanercept is a dimeric fusion protein consisting of the extracellular portion of the human p75 TNFR linked to the Fc portion of human $\lg \mathrm{g} 1 .^{26}$

To better serve as therapeutic agents, etanercept was designed to improve upon the characteristics of monomeric sTNFRs. Like naturally occurring sTNFRs, it is highly specific for TNF and lymphotoxin $\alpha$. However, compared with monomeric sTNFRs, etanercept has a much higher affinity for $\mathrm{TNF}{ }^{26}$ In addition, the presence of the Fc portion of human IgG extends the half life of etanercept approximately fivefold to eightfold in vivo.

\section{Clinical studies of sTNFR based agents in patients with RA}

Etanercept has been assessed in clinical trials of adults with RA and patients with juvenile RA (JRA). On the basis of extensive data from the etanercept clinical trials, this agent became the first biological response modifier approved by the FDA for use in patients with RA, and JRA.

\section{ETANERCEPT CLINICAL TRIALS}

The potential clinical utility of etanercept in adults with RA was assessed in three placebo controlled, double blind, randomised clinical trials involving over 500 patients. In the first placebo controlled etanercept trial, a phase II trial involving 180 patients who had failed treatment with from one to four disease modifying anti-rheumatic drugs (DMARDs), pa- tients received a subcutaneous injection of placebo or etanercept $0.25,2$, or $16 \mathrm{mg}$ per square metre of body surface area) twice weekly for three months. ${ }^{27}$ Etanercept produced a significantly greater improvement in all primary and secondary measures of disease activity than did placebo. A dose response relation was observed between etanercept dose and the primary outcome measures, with the greatest response from the $16 \mathrm{mg} / \mathrm{m}^{2}$ dose $\left(16 \mathrm{mg} / \mathrm{m}^{2}\right.$ is roughly equivalent to $25 \mathrm{mg}$ ). The mean reduction from baseline in total swollen and tender joint count was $61 \%$ with etanercept $16 \mathrm{mg} / \mathrm{m}^{2}$ and $25 \%$ with placebo (fig 1). The difference between the two groups was apparent as early as two weeks after starting treatment. At the end of the three month treatment period, a significantly larger percentage of patients in the etanercept $16 \mathrm{mg} / \mathrm{m}^{2}$ group $(75 \%)$ than in the placebo group (14\%) had at least a $20 \%$ improvement in the ACR. Similarly, $57 \%$ of patients receiving etanercept $16 \mathrm{mg} / \mathrm{m}^{2}$ experienced a $50 \%$ improvement in symptoms, compared with only $7 \%$ of the placebo group (a significant difference). Disease activity returned toward baseline within two months after discontinuation of etanercept treatment, suggesting the need for continued treatment.

On the basis of the favourable results from the phase II study, a second placebo controlled study was conducted using a simplified etanercept dosing schema and an extended duration of treatment. ${ }^{28}$ In this trial, 234 patients with refractory RA (inadequate response to 1-4 prior DMARDs) were randomised to receive placebo, etanercept $10 \mathrm{mg}$, or etanercept 25 mg subcutaneously twice weekly. All patients were required to have at least 12 tender/10 swollen joints, and at least one of the following: erythrocyte sedimentation rate $(\mathrm{ESR}) \geqslant 28$ $\mathrm{mm} 1 \mathrm{st} \mathrm{h}, \mathrm{C}$ reactive protein $(\mathrm{CRP})>2.0$ $\mathrm{mg} / \mathrm{dl}$, or morning stiffness for at least $45 \mathrm{~min}$ utes. The primary efficacy end points were $20 \%$ and $50 \%$ improvement in disease activity at three and six months, as defined in the ACR 

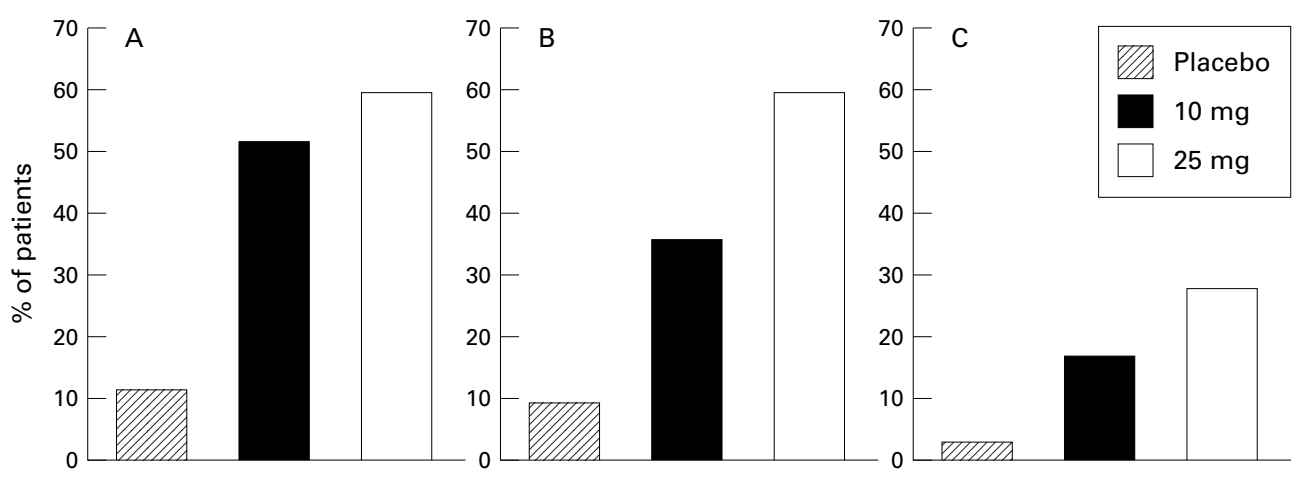

Figure 2 ACR responses at six months in phase III trial. (A) Per cent of patients achieving a 20\% ACR response. (B) Per cent of patients achieving a 50\% ACR response. (C) Per cent of patients achieving a $70 \%$ ACR response.

Table 1 Mean per cent improvement in quality of life at six months* (Phase III)

\begin{tabular}{llll}
\hline & & \multicolumn{2}{l}{ Etanercept } \\
\cline { 3 - 4 } Variable & $\begin{array}{c}\text { Placebo } \\
(n=80)\end{array}$ & $\begin{array}{l}10 \mathrm{mg} \\
(n=76)\end{array}$ & $\begin{array}{l}25 \mathrm{mg} \\
(n=78)\end{array}$ \\
\hline Disability Index & 2 & $34 \dagger$ & $39 \dagger$ \\
General health status & -12 & $34 \dagger$ & $33 \dagger$ \\
Arthritis specific health status & -22 & $31 \dagger$ & $44 \dagger$ \\
Vitality & 2 & $22 \dagger$ & $25 \dagger$ \\
Mental health & 3 & 17 & $35 \dagger \ddagger$ \\
\hline
\end{tabular}

^Positive numbers represent improvement, negative numbers represent worsening.

$t \mathrm{p}<0.05$ for etanercept groups compared with placebo group. $\neq \mathrm{p}<0.05$ for etanercept $10 \mathrm{mg}$ compared with etanercept 25 mg.

response criteria. Secondary end points included the individual components of the ACR response. This trial employed joint assessors who were blinded to treatment, were not involved in patient care, and did not discuss the study with patients or investigators.

Etanercept produced significantly greater improvement in all primary and secondary measures of disease activity than did placebo, and a dose response was apparent, with etanercept $25 \mathrm{mg}$ being more effective than etanercept $10 \mathrm{mg}$. At six months, $59 \%$ of patients in the etanercept $25 \mathrm{mg}, 51 \%$ of patients in the etanercept $10 \mathrm{mg}$, and $11 \%$ of patients in the placebo group achieved a $20 \%$ ACR response ( $p<0.001$ for each etanercept group compared with the placebo group). The percentage of patients achieving a $50 \%$ and $70 \%$ ACR response was also superior in patients receiving etanercept (fig $2 ; p \leqslant 0.001$ for etanercept 25 mg $v$ placebo).
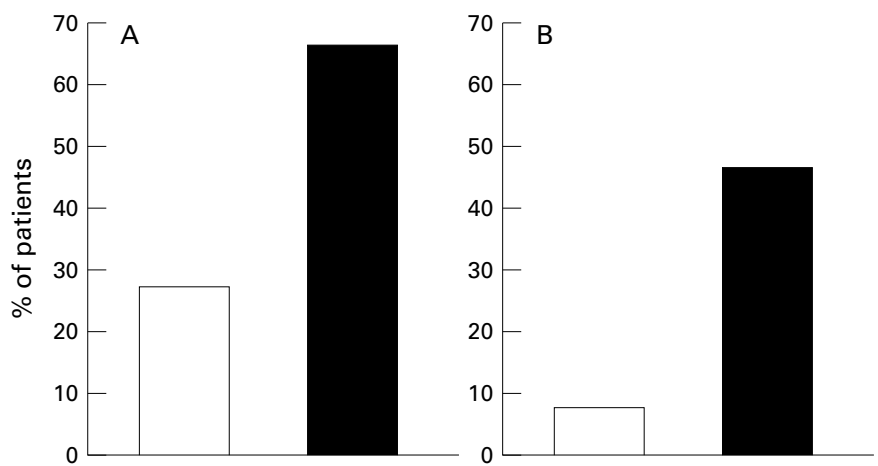

Figure 3 ACR responses at six months in methotrexate \pm etanercept trial. (A) Per cent of patients achieving a $20 \%$ ACR response. (B) Per cent of patients achieving a 50\% ACR response. (C) Per cent of patients achieving a $70 \%$ ACR response.

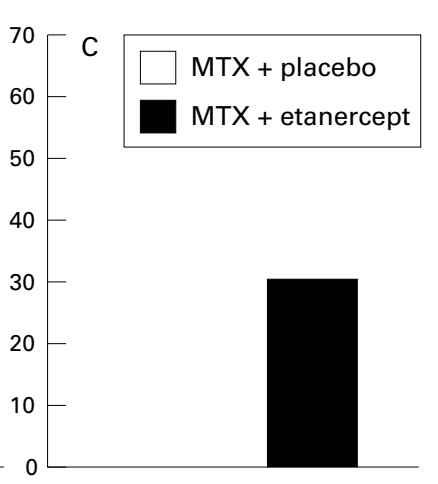

Clinical responses generally were observed within one or two weeks and nearly always within three months after starting treatment. Of patients receiving etanercept $25 \mathrm{mg}, 15 \%$ experienced a $70 \%$ ACR response after three months and six months of treatment, compared with fewer than $5 \%$ of patients who received placebo. The components of patient assessment of physical function (that is, disability index) measured using the Health Assessment Questionnaire were improved to a significantly greater extent by etanercept than by placebo (table 1 ).

In the third randomised etanercept trial in adult RA, the combination of methotrexate (MTX) and etanercept was compared with MTX plus placebo. ${ }^{29}$ In this double blind, randomised study, 89 patients with persistently active RA despite MTX treatment received subcutaneous injections of either placebo or etanercept $25 \mathrm{mg}$ twice weekly in addition to continuing MTX (12.5 to $25 \mathrm{mg}$ per week). The combination of etanercept and methotrexate resulted in $71 \%$ of patients achieving a $20 \%$ ACR response, compared with only $27 \%$ of patients who received methotrexate alone. As with the phase 3 trial, the percentage of patients achieving a $50 \%$ and $70 \%$ ACR response was statistically significantly greater for patients receiving etanercept (fig 3). Responses were rapid and durable.

The improvements in disease activity mediated by etanercept are maintained with long term treatment in the majority of patients (Moreland LW, et al. XIVth European Congress of Rheumatology, Glasgow, 1999). Of 51 


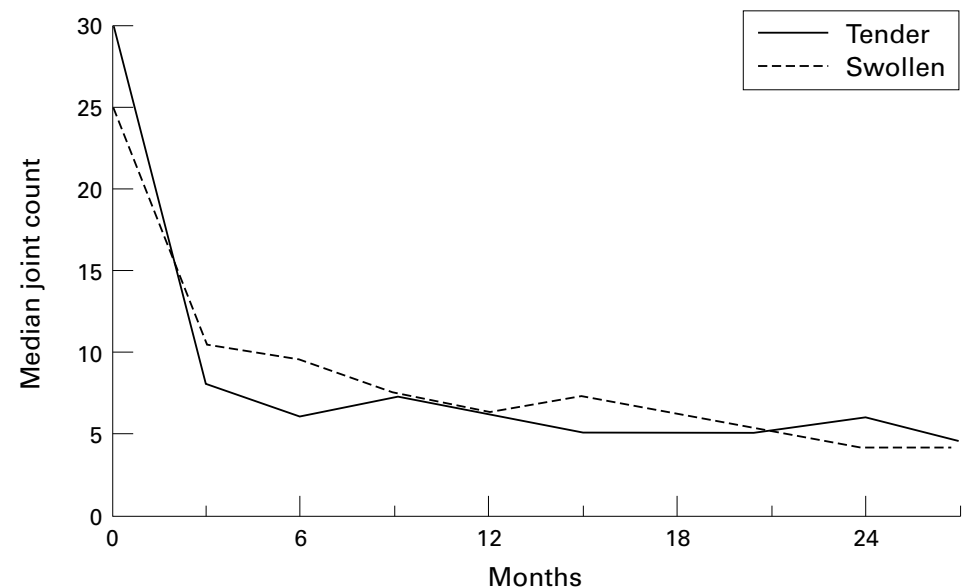

Figure 4 Median tender and swollen joint counts in long term open label etanercept trial.

patients treated with etanercept $25 \mathrm{mg}$ subcutaneously twice weekly for 24 months in a long term open label trial, tender and swollen joint counts were improved by 87 and $84 \%$, respectively, and $88 \%$ of patients achieved a Paulus 20 response at 24 months (fig 4 ).

Finally, preliminary results of a placebo controlled trial of etanercept in JRA have been reported (Lovell DJ, et al. American College of Rheumatology 62nd National Meeting, San Diego, 1998). Eligible patients were between the ages of 4-17 and had active, polyarticular course JRA. All patients received etanercept $0.4 \mathrm{mg} / \mathrm{kg}$ subcutaneously twice weekly for up to three months. Patients who met the JRA definition of improvement criteria at the end of three months were then randomised to continue on etanercept at the same dose and schedule or to receive placebo until disease flare occurred, or until four months elapsed, whichever was earlier. Sixty nine patients enrolled in the trial, and $51(74 \%)$ met the criteria for improvement at three months and entered the blinded portion of the trial. A significantly greater number of patients who received placebo had disease flare than did patients who received etanercept.

Data from these studies indicate that etanercept has a favourable safety profile. In placebo controlled trials, the discontinuation rate attributable to adverse events was similar in the etanercept and placebo groups. The most common etanercept associated adverse event is injection site reaction, which developed in 37\% of patients receiving etanercept versus $10 \%$ of those receiving placebo. This reaction is characterised by development of mild erythema, itching, pain, and/or swelling. The frequency of injection site reactions diminishes with time. In most RA patients these reactions do not necessitate drug discontinuation or require treatment.

Treatment with etanercept does not impair immunocompetence, as assessed by DTH skin testing, serum immunoglobulin concentrations, or enumeration of immune effector cell populations (Moreland LW, et al. American College of Rheumatology 62nd National Meeting). The overall incidence of infections and serious infections were similar in the etanercept and placebo groups in clinical trials. However, because data from clinical trials of etanercept in patients with microbial sepsis suggested an increase in mortality in patients receiving the highest dose $\left(1.4 \mathrm{mg} / \mathrm{kg}\right.$ body weight), ${ }^{30}$ etanercept should be used with caution in patients with serious infections, and these patients should be closely monitored.

Anti-etanercept antibodies were detected at least once in the sera of $16 \%$ of treated RA patients at multiple time points. However, this positive titre generally appeared only sporadically, and the antibodies were non-neutralising. Development of antibodies did not correlate to clinical response or adverse events. Treatment with etanercept has no clear effect on the development of autoantibodies and, no patients developed clinical signs suggestive of a lupus-like syndrome or other new autoimmune diseases during clinical trials. Etanercept does not cause liver or kidney damage and thus regular blood monitoring is not required, and no increase in the incidence of malignancy has been observed to date in etanercept treated patients.

\section{Future considerations}

TNF antagonism with etanercept has already become an important new therapy for the treatment of RA and JRA. The experience obtained to date in clinical trials leaves little doubt about the efficacy of etanercept in controlling symptoms in these patients. The question remains about the ability of etanercept to slow disease progression. Based on the preclinical data on TNF, etanercept would be expected to slow bone and cartilage destruction; however, previous trials have not investigated this issue. This question will soon be answered when the results of the largest randomised trial to date of etanercept in RA are reported. In this study, patients with early RA (less than three years since diagnosis and no prior methotrexate treatment) were randomised to receive etanercept $10 \mathrm{mg}$, etanercept $25 \mathrm{mg}$, or optimal doses of methotrexate for 12 months. Patients were evaluated clinically, as in previous trials, and radiographically, to determine the extent of progression of bony erosions. The results of this trial are likely to have a major impact on the ultimate place for etanercept in the treatment of RA. In addition, as more experience with etanercept is gained, effects on corticosteroid, NSAID, and DMARD requirements will probably become available, as well as information about long term health impact such as effects on disability and requirements for joint replacements.

The success of etanercept in RA will probably be carried over into other TNF mediated diseases. Not only does TNF play a part in other rheumatological conditions but also in Crohn's disease, heart failure, and numerous other inflammatory conditions. Controlling TNF represents an important new avenue by which many diseases may one day be treated.

1 Pennica D, Nedwin GE, Hayflick JS, et al. Human tumour necrosis factor: precursor structure, expression and homology to lymphotoxin. Nature 1984;312:724-9. 
2 Chu CQ, Field M, Feldmann M, Maini RN. Localization of tumor necrosis factor $\alpha$ in synovial tissues and at the cartilage-pannus junction in patients with

3 Bazzoni F, Beutler B. The tumor necrosis factor ligand and receptor families. N Engl J Med 1996;334:1717-25.

4 Jones EY, Stuart DI, Walker NP. Structure of tumour necrosis factor. Nature 1989;338:225-8.

5 Keffer J, Probert L, Cazlaris H, et al. Transgenic mice expressing human tumor necrosis factor: A predictive genetic model of arthritis. EMBO J 1991;10:4025.

6 Husby G, Williams RC Jr. Synovial localization of tumor necrosis factor in patients with rheumatoid arthritis. Autoimmun 1988;1:363-71.

7 Saxne T, Palladino MA Jr, Heinegård D, Talal N, Wollheim FA. Detection of tumor necrosis factor $\alpha$ but not tumor necrosis factor $\beta$ in rheumatoid arthritis synovial fluid and serum. Arthritis Rheum 1988;31:1041-5.

8 Tetta C, Camussi G, Modena V, Di Vittorio C, Baglioni C. Tumour necrosis factor in serum and synovial fluid of Tumour necrosis factor in serum and synovial fluid of patients with active and se
Rheum Dis 1990;49:665-7.

9 Hopkins SJ, Humphreys M, Jayson M1V. Cytokines in synovial fluid. 1 . The presence of biologically active and immunoreactive IL-1. Clin Exp Immunol 1988;72:422-7.

10 Tucci MA, Baker R, Mohamed A, Tsao AK, Hughes J Synovial tissues collected from rheumatoid patients undergoing total joint arthroplasty express markers for acute inflammation. Biomed Sci Instrum 1997;34:169-74. 11 Okamoto $\mathrm{H}$, Yamamura M, Morita Y, Harada S, Makino $\mathrm{H}$,
Ota Z. The synovial expression and serum levels of interleukin-6, interleukin-1 1, leukemia inhibitory factor, and oncostatin $\mathrm{M}$ in rheumatoid arthrits. Arthritis Rheum 1997;40:1096-105

12 Neidel J, Schuize M, Lindschau J. Association between degree of bone-erosion and synovial fluid-levels of tumor necrosis factor a in the knee-joints of patients with rheumatoid arthritis. Inflamm Res 1995;44:217-21.

13 Saklatvala J. Tumour necrosis factor (x stimulates resorption and inhibits synthesis of proteoglycan in cartilage. [Letter]. Nature 1986;322:547-9.

14 Bertolini DR, Nedwin GE, Bringman TS, Smith DD, Mundy GR. Stimulation of bone resorption and inhibition of bone formation in vitro by human tumour necrosis factors. Nature 1986;319:516-18.

15 Dayer J-M, Beutler B, Cerami A. Cachectin/tumor necrosis factor stimulates collagenase and prostaglandin E2production by human synovial cells and derma fibroblasts. J Exp Med 1985;162:2163-8.

16 Lader CS, Flanagan AM. Prostaglandin E2, Interleukin la, and tumor necrosis factor-a increase human osteoclast formation and bone resorption in vitro. Endocrinology 1998 ; 139:3157-64

17 Nawroth PP, Bank I, Handley D, Cassimeris J, Chess L, Stern D. Tumor necrosis factor/cachectin interacts with endothelial cell receptors to induce release of interleukin 1. J Exp Med 1986;163:1363-75.
18 Butler DM, Maini RN, Feldmann M, Brennan FM. Modulation of proinflammatory cytokine release in rheumatoid inovial membrane cell cultures. Comparison of monoclonal anti TNF-oc antibody with the interleukin-1 receptor antagonist. Eur Cytokine Netw 1995;6:225-30.

19 Brennan FM, Chantry D, Jackson A, Maini R, Feldmann M. Inhibitory effect of TNF $\alpha$ antibodies on synovial cell interleukin-1 production in rheumatoid arthritis. Lancet 1989;ii:244-7.

20 Brockhaus M, Schoenfeld H-J, Schlaeger E-J, Hunziker W, Lesslauer W, Loetscher H. Identification of two types of tumor necrosis factor receptors on human cell lines by monoclonal antibodies. Proc Natl Acad Sci USA 1990;87: 3127-31

21 Van Zee KJ, Kohno T, Fischer E, Rock CS, Moldawer LL, Lowry SF. Tumor necrosis factor soluble receptors circulate during experimental and clinical inflammation and can protect against excessive tumor necrosis factor $\alpha$ in and can protect against excessive tumor necrosis factor $\alpha$ in
vitro and in vivo. Proc Natl Acad Sci USA 1992;89:4845-9.

22 Cope AP, Aderka D, Doherty M, et al. Increased levels of soluble tumor necrosis factor receptors in the sera and synovial fluid of patients with rheumatic diseases. Arthritis Rheum 1992;35:1160-9.

23 Brennan FM, Gibbons DL, Cope AP, Katsikis P, Maini RN, Feldmann M. TNF inhibitors are produced spontaneously by rheumatoid and osteoarthritic synovial joint cell cultures: evidence of feedback control of TNF action. Scand J Immunol 1995;42:158-65.

24 Roux-Lombard P, Punzi L, Hasler F, et al. Soluble tumor necrosis factor receptors in human inflammatory synovial fluids. Arthritis Rheum 1993;36:485-9.

25 Barrera P, Boerbooms AMT, Janssen EM, et al. Circulating soluble tumor necrosis factor receptors, interleukin-2 receptors, tumor necrosis factor $\alpha$, and interleukin-6 levels in rheumatoid arthritis. Longitudinal evaluation during methotrexate and azathioprine therapy. Arthritis Rheum 1993;36:1070-9.

26 Mohler KM, Torrance DS, Smith CA, et al. Soluble tumor necrosis factor (TNF) receptors are effective therapeutic agents in lethal endotoxemia and function simultaneously agents in lethal endotoxemia and function simultaneously 1993;151:1548-61.

27 Moreland LW, Baumgartner SW, Schiff MH, et al. Treatment of rheumatoid arthritis with a recombinant human tumor necrosis factor receptor (p75)-Fc fusion protein. N Engl J Med 1997;337:141-7.

28 Moreland LW, Schiff MH, Baumgartner SW, et al. Etanercept therapy in rheumatoid arthritis. Ann Intern Med 1999;130:478-86.

29 Weinblatt ME, Kremer JM, Bankhurst AD, et al. A trial of etanercept, a recombinant tumor necrosis factor receptor:Fc fusion protein, in patients with rheumatoid arthritis receiving methotrexate. N Engl J Med 1999;340: 253-9.

30 Fisher CJJr, Agosti JM, Opal SM, et al. Treatment of septic shock with the tumor necrosis factor receptor:Fc fusion protein. N Engl J Med 1996;334:1697-702. 